\title{
Characterization of Grain-Oriented Electrical Steels Under High DC Biased Conditions
}

\author{
Christopher W. Harrison and Philip I. Anderson \\ Wolfson Centre for Magnetics, School of Engineering, Cardiff University, Cardiff CF24 3AA, U.K.
}

Power transformers are designed to operate under pure ac excitation; however, they may be subjected to superimposed dc voltages and consequential offset currents, for example, from geomagnetically induced currents, geomagnetically induced quasi-dc currents, arising from solar activity. To determine the effects of dc offsets, a single-sheet tester was used with a digital excitation signal created with an ac waveform summed with a dc voltage addition and measured with a digital feedback control algorithm to ensure an ideal flux density waveform. Using this method, results with $300 \%$ additional dc contribution were able to be measured for results up to $B_{\mathrm{ac}}+B_{\mathrm{dc}}=1.9 \mathrm{~T}$. The measurements of grain-oriented $3 \% \mathrm{Si}-\mathrm{Fe}$ show increasing power losses and peak applied magnetic field for peak-to-peak flux densities above $3 \mathrm{~T}$, with a $33 \%$ increase in power loss and $500 \%$ increase in applied magnetic field for an offset of $25 \%$.

Index Terms-DC bias, electrical steels, power losses.

\section{INTRODUCTION}

$\mathbf{P}$ OWER transformers are normally designed to operate with a sinusoidal input voltage. Increasing studies are being performed when the primary voltage becomes distorted; an example of this is due to the addition of a dc bias. A dc bias may arise in a transformer from many sources, such as unsymmetrical voltage levels due to ac-dc energy conversion and monopolar operation of HVdc [1], and solar activity may lead to geomagnetically induced quasi-dc currents (GICs) through the power transmission system and into the transformer through the grounded neutral terminal. An equivalent dc current of $25 \mathrm{~A}$ per phase has been observed for several hours from a dc injection of one of the ground electrodes of a HVdc. Comparatively, from GICs, 100 A per phase can be seen for up to $1 \mathrm{~min}$ and $50 \mathrm{~A}$ for up to $5 \mathrm{~min}$ [2]. These can lead to half-cycle saturation leading to a dramatic increase in excitation current, higher harmonic content, over heating (e.g., [3]-[5]), and noise levels [6]. This is illustrated in Fig. 1, which shows the potential for a small de offset to cause a power transformer to enter half-cycle saturation.

The magnetic properties of electrical steel are usually measured with a sinusoidal magnetic flux density at a fixed level of magnetic induction. Therefore, a preferred parameter for the dc biasing would be an additional constant component of the magnetic induction [7], [8]. In this paper, a method is introduced to measure the magnetic properties of electrical steel with up to a $300 \%$ dc offset of the magnetic flux density.

\section{EXPERIMENTAL PROCEDURE}

\section{A. Samples}

Measurements were performed on a single-sheet sample of $3 \%$ grain-oriented electrical steel M85-23 with

Manuscript received August 31, 2015; revised December 15, 2015; accepted January 12, 2016. Date of publication January 20, 2016; date of current version April 15, 2016. Corresponding author: C. W. Harrison (e-mail: harrisonc11@cardiff.ac.uk).

Color versions of one or more of the figures in this paper are available online at http://ieeexplore.ieee.org.

Digital Object Identifier 10.1109/TMAG.2016.2519945

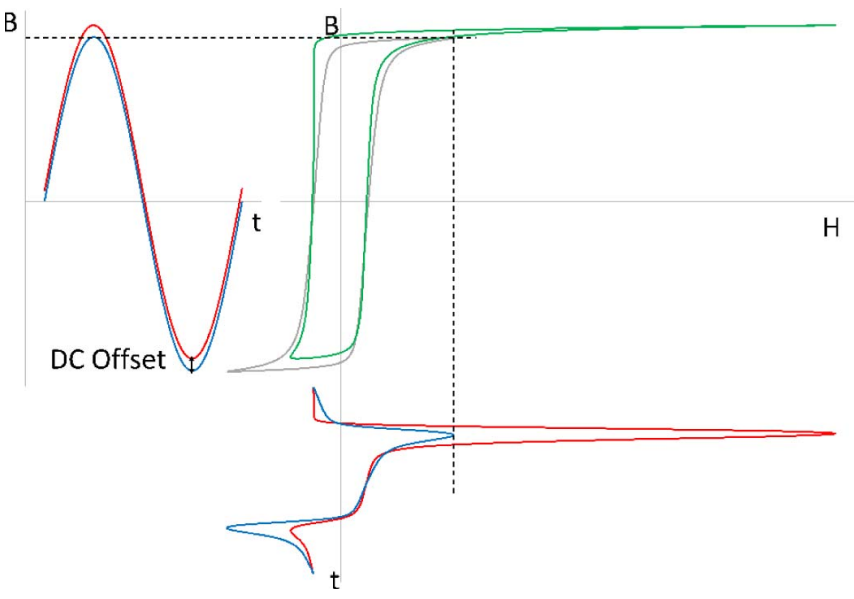

Fig. 1. Effect of the addition of a small dc offset to the magnetic flux density $B$ (left) may cause half-cycle saturation (right) and large distortions in the current waveform (bottom).

dimensions $100 \times 495 \mathrm{~mm}$ and mass $m=84.98 \mathrm{~g}$. All the measurements were performed in the rolling direction of the material. Initial measurements were performed with a pure sinusoidal flux density at $50 \mathrm{~Hz}$. The power losses at 1.5 and $1.7 \mathrm{~T}$ were 0.68 and $0.95 \mathrm{Wkg}^{-1}$, respectively.

\section{B. Measurement System}

Measurements were performed on a single-strip tester with flux closure around the sample provided by the laminated yokes of grain-oriented electrical steel. The total number of turns in the excitation coil and the search coil was 190 and 285, respectively; a compensation coil was also used. To apply a dc offset, an ideal digital flux density was generated. The makeup of this ideal waveform is shown in Fig. 2(c), which is a summation of a pure sinusoidal wave of five periods [Fig. 2(a)] and a trapezoidal wave [Fig. 2(b)], where the peak of the trapezoidal wave determined the amount of dc offset. Fig. 2(c) shows the second period highlighted from which the results in this paper were derived; in this case, an ac component of magnitude $B_{\mathrm{ac}}=0.5 \mathrm{~T}$ (peak to peak $1 \mathrm{~T}$ ) 

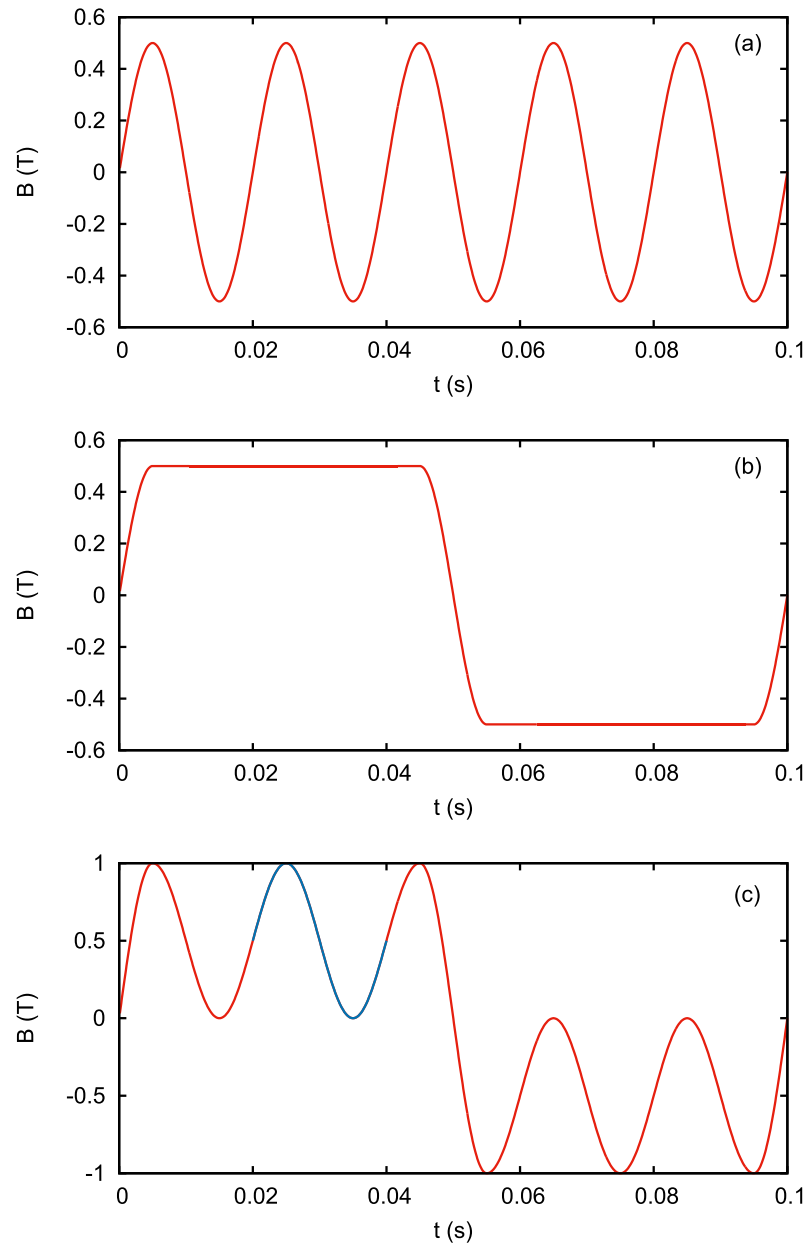

Fig. 2. Digital control waveform used for creating a dc offset. (a) and (b) are summed to create (c), with the highlighted waveform in (c) used in measurements.

and a dc offset of $100 \%$, with $B_{\mathrm{dc}}=0.5 \mathrm{~T}$. In all the results presented in this paper, the sinusoidal frequency component was $50 \mathrm{~Hz}$. In all the measurements with a dc offset, the magnetizing waveform was controlled with a digital feedback loop (as developed from the method described in [9]), where the difference in the ideal and measured $B$ and $d B / d t$ is iteratively used to modify the generated output voltage. A block diagram of the digital feedback system is shown in Fig. 3, showing the generated voltage passing through a digital low-pass filter to a power amplifier. The magnetizing current from the power amplifier is passed to the magnetizing coil through a shunt resistor, which allows current measurements. In addition, each iteration is measured over several periods and averaged, which increased the stability of the measurements. Average values between 5 and 10 were used in the measurements as this provided good stability while keeping the overall measurement time to within a few minutes. All the operations in the dc offset system were implemented in the software, and for integration (used to determine $B$ from the induced voltage), low-pass filtering (used in the digital feedback circuit) and Fourier transforms (used to derive total harmonic distortion) of the entire measured wave was used. This was done as the ideal wave function was symmetric and (a)
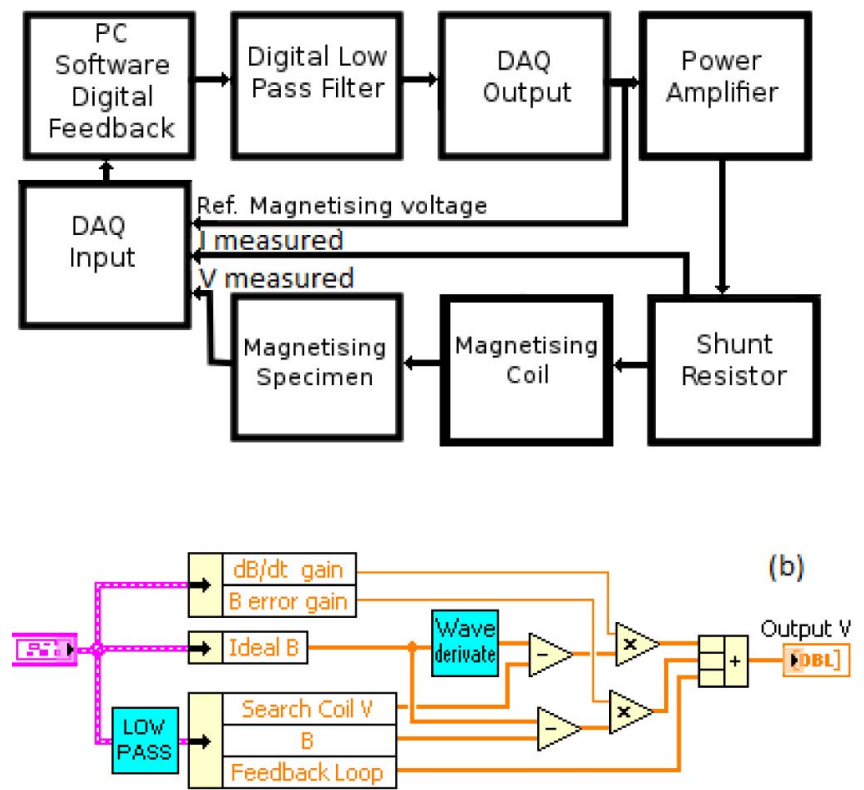

Fig. 3. (a) Block diagram of the digital feedback system. (b) Simplified LabVIEW program showing the process of the PC software digital feedback using the measured voltage, flux density, and reference magnetizing voltage (feedback loop).

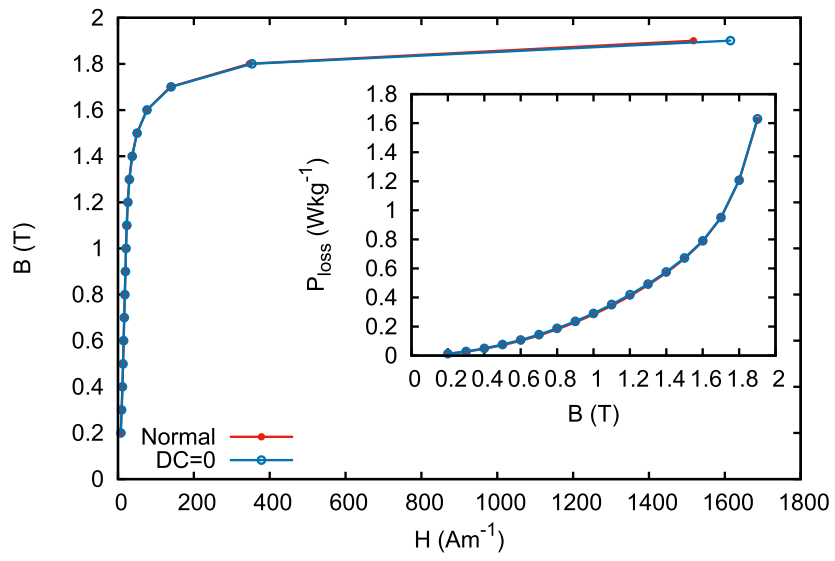

Fig. 4. Peak flux density $B$ as a function of applied field $H$ comparing normal measurement technique (normal) with the dc measurement technique with $B_{\mathrm{dc}}=0$. Inset: change in measured power loss.

could, therefore, be integrated without adding additional linear components. The total harmonic distortion was $<1 \%$, and the peak flux density value and form factor are within $0.1 \%$ of the ideal values in all the measurements. Total power losses were also numerically determined by

$$
P_{\text {Loss }}=\int_{0}^{T} H \cdot \frac{d B}{d t} d t .
$$

Fig. 4 shows a comparison between the magnetic properties of the standard measurement and the proposed method with a zero dc offset, with the main figure showing the $B-H$ curves and the inset the power losses. No differences in the $B-H$ curves or the power losses can be seen between the measurement techniques. 


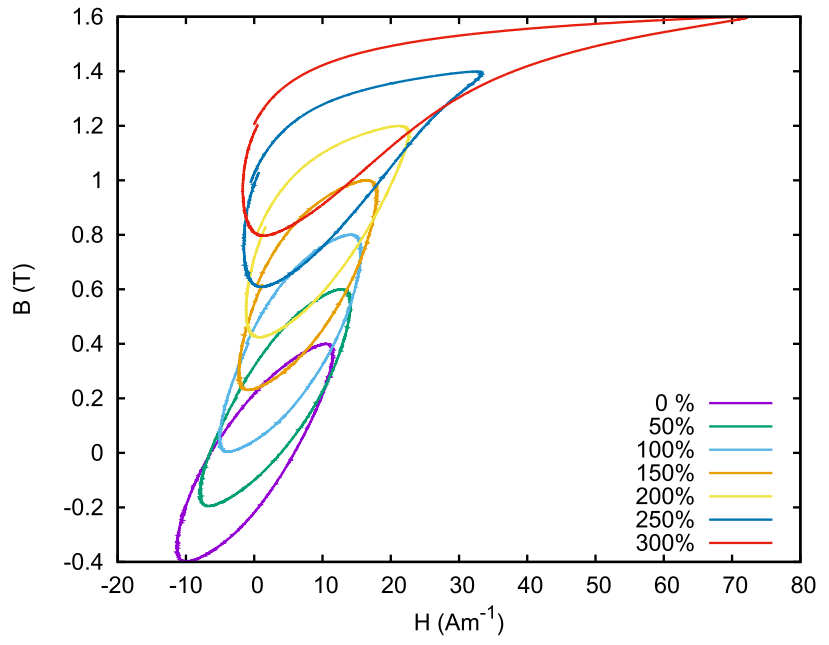

Fig. 5. Hysteresis loops of magnetic flux density $B$ as a function of applied magnetic field $H$ for varying amount of dc offsets.

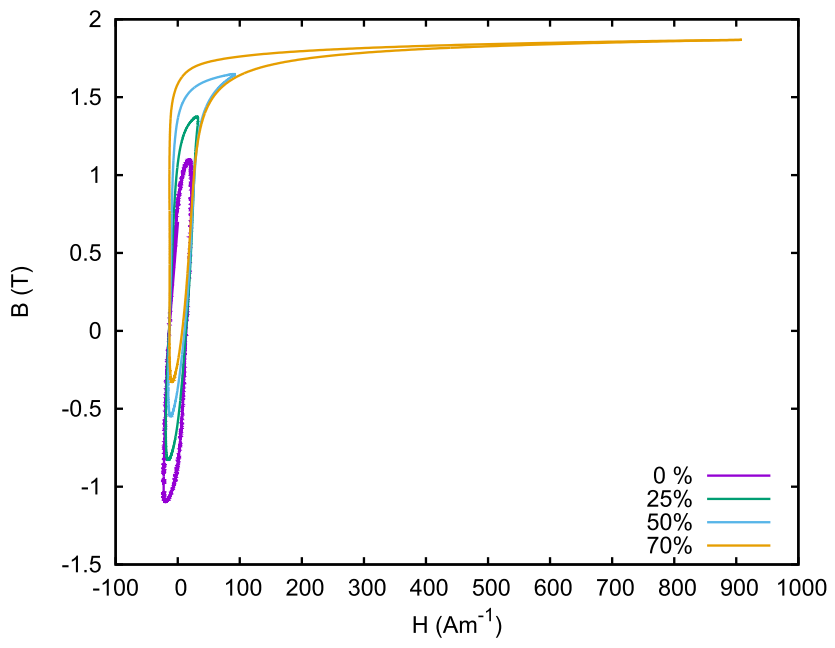

Fig. 6. Hysteresis loops of magnetic flux density $B$ as a function of applied magnetic field $H$ for varying amount of dc offsets.

\section{RESUlTS AND Discussion}

The magnetic properties were measured for the increasing values of dc offset from $0 \%$ to $300 \%$ and up to a total peak flux density $B=B_{\mathrm{ac}}+B_{\mathrm{dc}}=1.9 \mathrm{~T}$. An example of the $B-H$ loop for peak $B_{\mathrm{ac}}=0.4 \mathrm{~T}$ (peak to peak $B=0.8 \mathrm{~T}$ ) is shown in Fig. 5 and for $B_{\mathrm{ac}}=1.1 \mathrm{~T}$ (peak to peak $B=2.2 \mathrm{~T}$ ) in Fig. 6, showing the effect on the loop shape and the area for varying dc offsets. In Fig. 7, the maximum applied field $H_{\text {peak }}$ is shown for varying values of $B_{\mathrm{dc}}$. The value of $H_{\text {peak }}$ increases with increasing $B_{\mathrm{dc}}$, with a $500 \%$ increase in $H_{\text {Peak }}$ observed with $B_{\mathrm{dc}}=0.17$ and $0.3 \mathrm{~T}$ for $B_{\mathrm{ac}}=1.7$ and $1.5 \mathrm{~T}$, respectively. By comparing the results of Figs. 7 with 4 , the value of $H_{\text {peak }}$ at $B_{p}\left(=B_{\mathrm{ac}, p}+B_{\mathrm{dc}, p}\right)$ can be directly related to $B_{p}$ of the zero dc offset. This could allow for extrapolation beyond $B=1.9 \mathrm{~T}$ of these measurements [10] allowing the study of saturation values observed during the GIC events. The total power losses for all the measured dc offsets are shown in Fig. 8. The total power losses for several fixed peak-to-peak flux densities are shown in Fig. 9. At high peak-to-peak

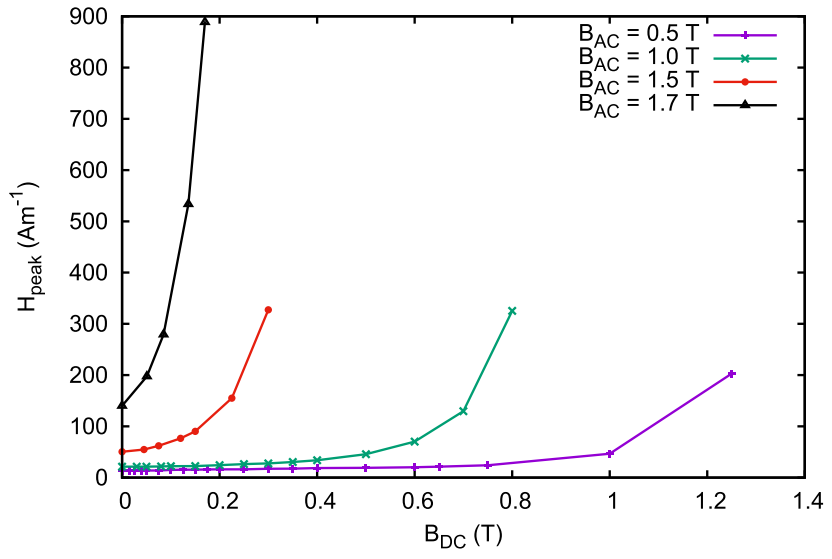

Fig. 7. Peak applied field $H_{\text {peak }}$ for varying applied dc offsets $B_{\mathrm{dc}}$. Results are shown for fixed peak sinusoidal flux densities $B_{\mathrm{ac}}$.

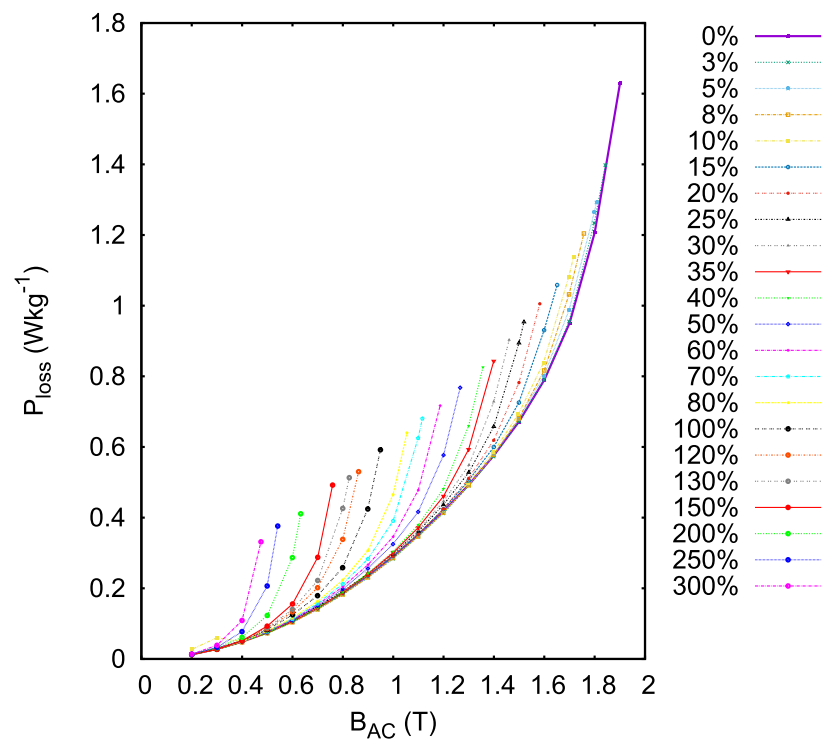

Fig. 8. Total power losses as a function of peak sinusoidal flux density $B_{\mathrm{ac}}$ for varying dc offset percentages.

ac flux densities, even a small addition of dc component can greatly increase the power losses by $10 \%$ dc increasing losses by $0.13 \mathrm{Wkg}^{-1}$ or $13 \%$ at $B_{\mathrm{ac}}=1.7 \mathrm{~T}$. At $B_{\mathrm{ac}}=1.5 \mathrm{~T}$, at $10 \%$ dc offset, the increase in losses is only $0.02 \mathrm{Wkg}^{-1}$ or $4 \%$. As the $\mathrm{dc}$ offset increases to $25 \%$, the increase in power losses increases to $0.22 \mathrm{Wkg}^{-1}$ or a change in $33 \%$. At low peak-to-peak flux densities of $B_{\mathrm{ac}}=0.4$ and $0.5 \mathrm{~T}$, no discernible change in power loss is seen below a dc field of $B_{\mathrm{dc}}=1 \mathrm{~T}$. This relates to a $200 \%$ and $250 \% \mathrm{dc}$ offset.

The effect on power loss with increasing dc bias shows a similar trend to previous measurements [7], [8], [11] when the offset is applied to the magnetic flux density. From Fig. 7, to determine a dc offset in the applied magnetic field at $200 \mathrm{Am}^{-1}, B_{\mathrm{ac}}=0.7 \mathrm{~T}$ shows a $100 \%$ increase in power loss, whereas at $B_{\mathrm{ac}}=1.7 \mathrm{~T}$, the losses only increase by $2 \%$. Results where the dc biasing is to the applied magnetic field [12] have shown similar results with a $100 \%-200 \%$ increase in power losses at $B_{\mathrm{ac}}=0.7 \mathrm{~T}$ for $H_{\mathrm{dc}}=100-400 \mathrm{Am}^{-1}$ and as peak-to-peak flux densities 


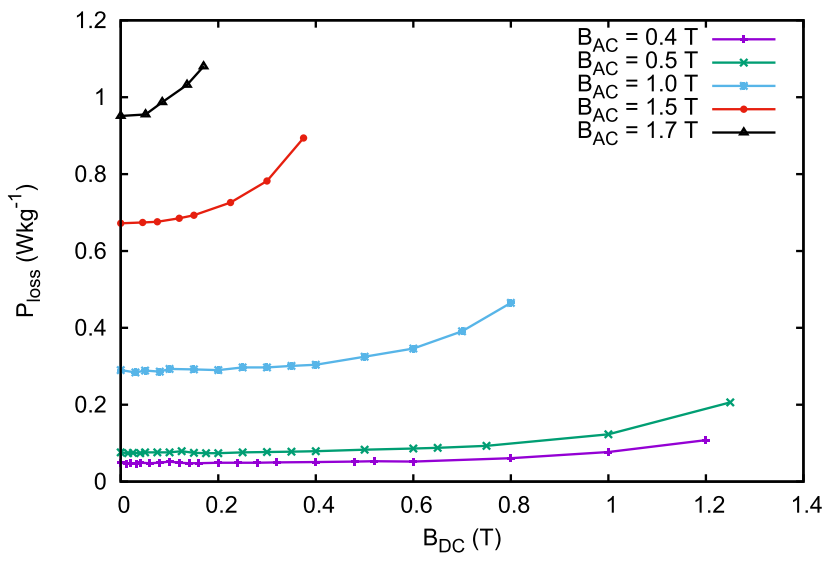

Fig. 9. Total power losses for varying applied dc offsets $B_{\mathrm{dc}}$. Results are shown for fixed peak sinusoidal flux densities $B_{\mathrm{ac}}$.

approach saturation; the difference between no dc and applied dc power losses reduces to $10 \%$ or less.

\section{CONCLUSiON}

A measurement system has been presented to allow the measurements of sinusoidal magnetic flux densities with a dc component of up to $300 \%$ for the measurement of magnetic steel sheets. The technique has been used to show that consideration need to be made for systems operating above $1.5 \mathrm{~T}$, where the dc offsets of the order of $25 \%$ can cause large additions to the power losses and large increases to the magnetizing current.

\section{ACKNOWLEDGMENT}

This work was supported by the National Grid.

\section{REFERENCES}

[1] D. Tousignant, L. Bolduc, and A. Dutil, "A method for the indication of power transformer saturation," Electr. Power Syst. Res., vol. 37, no. 2, pp. 115-120, May 1996. [Online]. Available: http://www.sciencedirect.com/science/article/pii/0378779696010462

[2] P. Picher, L. Bolduc, A. Dutil, and V. Q. Pham, "Study of the acceptable DC current limit in core-form power transformers," IEEE Trans. Power Del., vol. 12, no. 1, pp. 257-265, Jan. 1997.

[3] T. S. Molinski, "Why utilities respect geomagnetically induced currents," J. Atmos. Solar-Terrestrial Phys., vol. 64, no. 16, pp. 1765-1778, Nov. 2002. [Online]. Available: http://www.sciencedirect.com/science/ article/pii/S1364682602001268

[4] O. Bíró, G. Buchgraber, G. Leber, and K. Preis, "Prediction of magnetizing current wave-forms in a three-phase power transformer under DC bias," IEEE Trans. Magn., vol. 44, no. 6, pp. 1554-1557, Jun. 2008.

[5] S. Mousavi, G. Engdahl, and E. Agheb, "Investigation of GIC effects on core losses in single phase power transformers," Arch. Elect. Eng., vol. 60, no. 1, pp. 35-47, Mar. 2011.

[6] F. Bachinger et al., "Direct current in transformers: Effects and compensation," in Proc. CIGRE, 2012, pp. 1-5.

[7] S. Takada, T. Yasuda, and T. Sasaki, "Magnetizing properties of an oriented $\mathrm{Si}-\mathrm{Fe}$ sheet core in biased magnetizing states at power frequencies," J. Magn. Magn. Mater., vols. 157-158, pp. 455-456, May 1996. [Online]. Available: http://www.sciencedirect.com/science/ article/pii/0304885395012540

[8] P. Marketos, A. J. Moses, and J. P. Hall, "Effect of dc voltage on ac magnetisation of transformer core steel," J. Elect. Eng., vol. 61, no. 7/s, pp. 123-125, 2010.

[9] S. Zurek, P. Marketos, T. Meydan, and A. J. Moses, "Use of novel adaptive digital feedback for magnetic measurements under controlled magnetizing conditions," IEEE Trans. Magn., vol. 41, no. 11, pp. 4242-4249, Nov. 2005.

[10] A. E. Umenei, Y. Melikhov, and D. C. Jiles, "Models for extrapolation of magnetization data on magnetic cores to high fields," IEEE Trans. Magn., vol. 47, no. 12, pp. 4707-4711, Dec. 2011.

[11] E. Barbisio, O. Bottauscio, M. Chiampi, and C. Ragusa, "Analysis of AC magnetic properties in SiFe laminations under DC-biased magnetisation," Phys. B, Condensed Matter, vol. 343, nos. 1-4, pp. 127-131, Jan. 2004. [Online]. Available: http://www.sciencedirect. com/science/article/pii/S0921452603005647

[12] Z. Zhao et al., "Magnetic property modelling of laminated silicon steel sheets under DC-biasing magnetization," in Proc. 6th Int. Conf. Electromagn. Field Problems Appl. (ICEF), Jun. 2012, pp. 1-4. 